Bio-grafia Escritos sobre la Biologia y su Enseñanza.

Edición Extra-Ordinaria. ISSN 2027-1034 P.p183-195

Memorias del VII Encuentro Nacional de Experiencias en la Enseñanza de la

Biología y la Educación Ambiental y II Congreso Nacional de Investigación en la Enseñanza de la Biología

\title{
EL CONTEXTO COMO PUNTO DE PARTIDA EN UNA PROPUESTA DE EDUCACIÓN AMBIENTAL PARA EL CONOCIMIENTO Y CONSERVACIÓN DE LA AVIFAUNA EN LA LAGUNA DE PEDROPALO (TENA, CUNDINAMARCA) - COLOMBIA
}

\section{THE CONTEXT AS THE STARTING POINT ON ENVIRONMENTAL EDUCATION PROPOSALS TO CONSERVATION AND KNOWLEDGE TO THE AVIFAUNA AT THE PEDROPALO'S LAGOON (TENA, CUNDINAMARCA) - COLOMBIA}

\section{Astrid Mireya Castellanos Millán ${ }^{1}$ \\ Héctor Edwin Beltrán Gutiérrez ${ }^{2}$}

\section{Resumen.}

Se diseñó y aplicó una encuesta a los adultos acudientes y estudiantes de los grados tercero, cuarto y quinto de la Institución Educativa Departamental Betulia, sede Catalamonte, única en el área de influencia de la Reserva Laguna de Pedropalo (Tena-Cundinamarca), esta últimade alto valor ornitológico, símbolo de pertenencia y fuente de recursos. Con el objeto de hacer una lectura de contexto, que dé cuenta de las interrelaciones físicas, biológicas, sociales y culturales que teje la comunidad con los demás humanos y seres vivos, complementadas con análisis de documentos, anotaciones y diarios de campo. Los resultados evidenciaron la importancia de indagar aspectos de orden personal, académico, social y ambiental de la cotidianidad de los estudiantes y sus acudientes, a través de actitudes y aptitudes en el desarrollo del cuestionario y de manifestar oralmente sus percepciones, inquietudes e inconformidades. Así, la lectura de contexto es fundamental para comprender la realidad de los colectivos implicados en problemáticas ambientales que permitan el diseño, planeación y ejecución de estrategias significativas frente al conocimiento y conservación de la avifauna, a través de iniciativas en Educación Ambiental.

Palabras Clave: contexto, encuesta, Educación Ambiental, conservación

\section{Abstract.}

\footnotetext{
${ }^{1}$ Licenciada en Biología Universidad Distrital Francisco José de Caldas. Estudiante Especialización en Educación y Gestión Ambiental. E-mail: licenbioastridcm@gmail.com

${ }^{2}$ Licenciado en Biología Y Magister en ciencias Biológicas. Profesor T.C. Universidad Distrital Francisco José de Caldas. E-mail: hebeltran@udistrital.edu.co
}

Este artículo es producto del proyecto de investigación en pregrado, titulado: Vinculación de la comunidad escolar a través de estrategias didácticas al conocimiento y conservación de la avifauna en la Laguna de Pedropalo (Tena-Cundinamarca), realizado al interior del Grupo de Investigación en Calidad Ambiental de la Universidad Distrital Francisco José de Caldas. 
Memorias del VII Encuentro Nacional de Experiencias en la Enseñanza de la

Biología y la Educación Ambiental y II Congreso Nacional de Investigación en la

\section{Enseñanza de la Biología}

The present research consisted of designing and applying a poll for elementary grade students and their parents in the Departmental Educational Institution Betulia, Catalamonte. This institution is the only one located at the influence area of Pedropalo's Lagoon Reserve (Tena-Cundinamarca) and it is considered a place of high biological potential, a belonging symbol and a significant source of natural resources. The objective was the application of the poll for developing a reading of context to highlight the physical, biological, social and cultural interrelationship behaviors of all the community with others human and living beings, supplemented by document analysis, notes and field diaries. The results of the survey show attitudes, skills, perceptions and disagreements of the students and their parents, regarding aspects of their personal, academic, social and environmental behavior, allowing them to interact with their environment. Therefore, the reading context is essential to understand the reality of the groups involved in environmental issues to enable the design, planning and implementation of strategies towards knowledge and significant bird conservation through environmental education initiatives.

Keywords: context, poll, Environmental Education, conservation

\section{Introducción}

La Reserva Forestal Protectora Laguna de Pedropalo, se ubica en el municipio de Tena (Cundinamarca-Colombia) y se encuentra dentro del Área de Importancia para la Conservación de las Aves (AICA) Bosques de la Falla del Tequendama (Freile y Santander, 2005), como un sitio críticamente importante a nivel mundial para las aves y la biodiversidad (BirdLife, 2005), con cerca de 202 especies de aves reportadas, algunas endémicas o casi endémicas y en alguna categoría de amenaza (Freile y Santander, 2005). La problemática de la reserva Laguna de Pedropalo, se centra principalmente en la tala de bosques, la implementación de sistemas agrícolas, ganaderos y de producción maderera, también, el crecimiento de la población, lo que genera destrucción, fragmentación de hábitats y cacería (Renjifoet al., 2005).

De acuerdo con la Ley 1549/2012, para permitir la participación activa de los individuos y sus comunidades en pro de la transformación de su realidad, la Educación Ambiental debe ser entendida, como un proceso dinámico y participativo, orientado a la formación de personas críticas y reflexivas, con capacidades para comprender las problemáticas ambientales de sus contextos (locales, regionales y nacionales), éstos últimosentendidos como sistemas, a través de los cuales se desarrollan las diversas dinámicas socioculturales, que dan cuenta de las interacciones entre los espacios físicos, geográficos, ecológicos y/o ambientales y la comunidad, pero también donde los individuos y los colectivos, se reconocen y reconocen a los demás. Entonces, en la construcción de escenarios para la Educación Ambiental y en la puesta en marcha de propuestas ambientales, 
Memorias del VII Encuentro Nacional de Experiencias en la Enseñanza de la

Biología y la Educación Ambiental y II Congreso Nacional de Investigación en la

$$
\text { Enseñanza de la Biología }
$$

se hace indispensable la lectura de contexto como el planteamiento de reflexiones fundamentales que permitan comprender las interacciones: sociedad-naturaleza y sus proyecciones en las diversas dinámicas culturales locales y/o regionales (Torres, 2002)

Así, debido a que la Reserva Laguna de Pedropalo es un hábitat representativo para especies de aves que tolera graves impactos antrópicos, se hace necesario reconocer el contexto desde y para lo ambiental, como un diálogo permanente entre la naturaleza la sociedad y la cultura, ésta lectura implica indagar sobre elentorno de estudiantes y sus acudientes, a través de la encuesta como método cuyos elementos: preguntas y respuestas son instrumentos importantes para la lectura de realidades ambientales (Peredaet al., 2003), dándole al presente trabajo un enfoque metodológico y fundamentación epistemológica de orden explicativo, cualitativo y descriptivo (Pérez, 2004).

Los aportes de la realización del presente trabajo fueron a las interrelaciones (conocimiento previo y comportamiento) que establecen los estudiantes y sus familias con las aves, lo que sirvió de base para el diseño e implementación de estrategias y herramientas didácticas para el conocimiento y conservación de la avifauna.

\section{Metodología}

La investigación tiene un carácter mixto (cualitativo-cuantitativo) en el marco de un enfoque metodológico de Investigación-Acciónen donde los estudiantes se involucran en la realización de las actividades, también en la actuación grupal donde colaboran en las diferentes fases del proceso de investigación (Goyete y Lessard, 1988).

Por consiguiente, se tomaron elementos de las técnicas conversacionales de Encuesta y Entrevista, la primera útil para dar cuenta de las razones u opiniones que tienen las personas acerca de determinados temas y la segunda, importante para conocer la perspectiva de los actores sociales (Sautu et al., 2005), por lo que se constituyó aquí un instrumento con características de "entrevista estructurada" (Corbetta, 2003) aplicable en el estudio de representaciones sociales personalizadas (normas, estereotipos) (Delgado y Gutiérrez, 1999).

De acuerdo con lo anterior, se generó un cuestionario estandarizado dirigidoque diera cuenta de manera directa o indirecta de la relación existente con distintas variables (Corbetta, 2003) del contexto escolar, las interrelaciones con la avifauna y su relación con la problemática ambiental tanto de estudiantes como de acudientes, pues de acuerdo con Torres (2002) sus elementos (preguntas y respuestas) tienen gran valor como herramientas de apropiación de la dinámica 
Memorias del VII Encuentro Nacional de Experiencias en la Enseñanza de la

Biología y la Educación Ambiental y II Congreso Nacional de Investigación en la

\section{Enseñanza de la Biología}

natural para los colectivos. Dicho test abarcó aspectos de carácter natural y sociocultural, cuyas respuestas fueron agrupadas, cuantificadas y representadas en gráficos de torta y que tuvieron como apoyo la explicación de un entrevistador con el fin de favorecer la producción de un discurso conversacional contínuo, con una cierta línea argumental del entrevistado sobre un tema definido (Corbetta, 2007).Así, para que no fuese una técnica cerrada limitada a cuantificar, fue necesario conocer y delimitar las posibles problemáticas ambientales de individuos o colectivos a través de consulta bibliográfica previa, observación y que el entrevistador informara e involucrara al entrevistado durante la aplicación (Peredaet al., 2003).

Por lo tanto, leer e interpretar el contexto es poner endiálogo a la naturaleza con la sociedad y la cultura, y por ende conocer los principales núcleos problémicos que dinamizan el proceso de construcción contextual (Torres, 2002), entre los que se encuentran aspectos: Físicos y de población, Biofísicos, Socioculturales y Económicos, razón por la cual la información de la percepción de dichos aspectos, según sus acciones y formas de interpretar su cotidianidad, se obtuvo a través de preguntas agrupadas en las siguientes categorías para padres de familia 0 acudientes:

\begin{tabular}{|c|c|}
\hline CATEGORİAS & PREGUNTAS RELACIONADAS \\
\hline $\begin{array}{l}\text { Personal. Categoría orientada a } \\
\text { conocer aspectos de orden personal } \\
\text { del acudiente del estudiante, pues el } \\
\text { ambiente familiar y las relaciones } \\
\text { interpersonales entre los individuos } \\
\text { responsables, son las bases del } \\
\text { desarrollo de los niños y niñas, } \\
\text { convirtiéndose así en las directrices } \\
\text { de su comportamiento }\end{array}$ & $\begin{array}{l}\text { Nombre de quién responde la encuesta, } \\
\text { se trata de la Madre/Padre/Otro. } \\
\text { - ¿Sabe leer y escribir? } \\
\text { - ¿Quiénes son sus hijos y las edades } \\
\text { de los mismos? } \\
\text { - ¿Vive con su cónyuge o pareja } \\
\text { sentimental? } \\
\text { - ¿Él o ella son el padre o la madre de } \\
\text { sus hijos? }\end{array}$ \\
\hline $\begin{array}{l}\text { Social. Categoría orientada a } \\
\text { determinar aspectos a nivel } \\
\text { económico como labor o actividad de } \\
\text { sustento, tipo de vivienda y } \\
\text { ubicación, y cobertura de servicios } \\
\text { públicos. Aspectos fundamentales }\end{array}$ & $\begin{array}{l}\text {-¿Qué tipo de labor desempeña? } \\
\text { Empleado (hoteles, ¿Qstaurantes, } \\
\text { cuidador)/Agricultura } \\
\text { cultiva?/Ganadería/Servicio } \\
\text { Doméstico/Industria } \\
\text { comercio/Construcción/Otro ¿Cuál? } \\
\text {-¿Su vivienda es propia o en arriendo? } \\
\text {-¿En qué vereda está ubicada su casa? } \\
\text {-¿Cuántas personas viven en la casa? } \\
\text {-¿Con cuáles servicios cuenta su } \\
\text { vivienda? } \\
\text { Energía/Acueducto/Alcantarillado/Teléfo } \\
\text { no/Gas/Recolección de basuras. }\end{array}$ \\
\hline
\end{tabular}


Memorias del VII Encuentro Nacional de Experiencias en la Enseñanza de la Biología y la Educación Ambiental y II Congreso Nacional de Investigación en la Enseñanza de la Biología

para establecer si los estudiantes trabajan y la forma de utilización de recursos físicos.
-Si no cuenta con alguno de los servicios anteriores ¿Cuál de estas actividades alternativas realiza? Cocina con leña/Se abastece de agua de ríos, quebradas o nacimientos de pozos sin bomba/Quema, entierra basura la deja a cielo abierto/Recicla

Salud. Categoría orientada a conocer la prestación de servicios de salud de los encuestados.

Educación. Categoría orientada a indagar el nivel educativo de los encuestados.
- ¿Está afiliado actualmente a una EPS o a Sisbén?

-¿En cuál centro lo atienden?

- ¿En qué nivel educativo se encuentra?

Primaria incompleta/secundaria completa/secundaria incompleta/técnico, profesional

tecnológico,
Ambiental. Categoría orientada a conocer la forma en que los encuestados conciben y se relacionan con los recursos naturales.
-¿Cuáles son para usted los recursos naturales más representativos? - ¿Considera que realiza actividades que involucren estos recursos? ¿Cuáles?

Tabla 1. Categorías y preguntas relacionadas para conocer el contexto de los adultos acudientes

Para una aproximación al concepto de ambiente construido por los estudiantes, fue necesario considerar preguntas que dieran cuenta de instrumentos físicos, sociales y simbólicos (Ángel, 1995) que incluyan actitudes, valores y creencias en sus contextos como espacios en los que se generan criterios de identidad derivados de sus sentidos de pertenencia, ligados a sus formas de interpretación del mundo, significación de fenómenos, construcción de imaginarios colectivos y de un mundo simbólico (Ley 1549/2012) a través de las siguientes categorías y preguntas:

\begin{tabular}{|l|l|}
\hline \multicolumn{1}{|c|}{ CATEGORÍAS } & \multicolumn{1}{|c|}{ PREGUNTAS RELACIONADAS } \\
\hline $\begin{array}{l}\text { Académico. Categoría } \\
\text { relacionada con el reconocimiento } \\
\text { de los estudiantes en el ámbito } \\
\text { escolar. }\end{array}$ & $\begin{array}{l}\text { ¿A qué grado perteneces? ¿Eres nuevo o } \\
\text { la misma escuela? ¿Cuántos? }\end{array}$ \\
\hline $\begin{array}{l}\text { Personal. Categoría orientada a a } \\
\text { conocer aspectos puntuales de los } \\
\text { estudiantes y de sus relaciones }\end{array}$ & $\begin{array}{l}\text { ¿Cuántos años tienes? ¿Trabajas? Todos } \\
\text { con tus padres? Con los dos/sólo con uno }\end{array}$ \\
\hline
\end{tabular}


Memorias del VII Encuentro Nacional de Experiencias en la Enseñanza de la

Biología y la Educación Ambiental y II Congreso Nacional de Investigación en la Enseñanza de la Biología

\begin{tabular}{|c|c|}
\hline $\begin{array}{l}\text { con las personas que los rodean } \\
\text { (familiares y compañeros). }\end{array}$ & ¿Cuál?/Sin mis padres \\
\hline $\begin{array}{l}\text { Ambiental. Categoría orientada a } \\
\text { conocer la forma en que los } \\
\text { estudiantes conciben y se } \\
\text { relacionan con los recursos } \\
\text { naturales. }\end{array}$ & $\begin{array}{l}\text { ¿Qué es para ti medio ambiente? ¿Cuáles } \\
\text { son los recursos naturales más } \\
\text { representativos? ¿Haces cosas buenas por } \\
\text { la conservación del medio ambiente? } \\
\text { ¿Cuáles? }\end{array}$ \\
\hline
\end{tabular}

Tabla 2. Categorías y preguntas relacionadas para conocer el contexto de los estudiantes.

Así,el cuestionario fue diseñado, aplicado y complementado por otras actividades (dilemas morales, mapas ambientales, herramientas de reconocimiento e identificación de la avifauna) a 19 de 21 estudiantes de los grados 3ㅜㅜ 4ํㅜ y 5o de la Institución Educativa Departamental Betulia sede Catalamonte única en el área de reserva de la Laguna de Pedropalo, en dos jornadas estudiantiles semanales durante los meses de febrero a mayo del 2009; y a 11 padres de familia, de forma directa por el investigador y con ayuda del profesorado.

\section{Resultados Y Discusión}

En la lectura de contexto para padres de familia y/o acudientes, los resultados de las encuestas fueron tabulados y representados en gráficos de torta, este diagnóstico indicó que el $19 \%$ de las personas que responden la encuesta son Padres, el $73 \%$ son Madresy el $9 \%$ de la muestra son abuelos u otros familiares, excepto un caso a cargo del ICBF. Las madres afirman vivir con su pareja sentimental $(73 \%)$, mientras el $27 \%$ son padres o madres que no viven con su compañero permanente. El $54 \%$ de las madres encuestadas tienen de 2 a 3 hijos, siendo éste el intervalo más frecuente, el $46 \%$ restante tienen uno, cuatro o cinco hijos. El $60 \%$ de la muestra afirma que su cónyuge es el padre o madre de los mismos, mientras el $40 \%$ vive con compañeros sentimentales diferentes a los padres biológicos. De acuerdo con lo anterior, conocer los aspectos indagados en la categoría de orden personal, son importantes pues el hogar y la unión familiar son factores determinantes en los procesos de aprendizaje, de modo que variables como la presencia de ambos padres en el hogar y el número de hermanos, afectarían al grado de seguimiento y control del proceso educativo del hijo, así la componente afectiva es esencial en la configuración de todo tipo de actitudes, y de aptitudes, incluidas las intelectuales (Mediavilla y Calero, 2009).

En la categoría social, el $46 \%$ de los encuestados vive de la agricultura o cultivo de pequeñas parcelas, donde se cultiva café, plátano, maíz, caña de azúcar, yuca y pastos, de manera artesanal. Son actividades no representativas económicamente, más bien de subsistencia, el $9 \%$ son empleados y otro $9 \%$ viven 
Memorias del VII Encuentro Nacional de Experiencias en la Enseñanza de la

Biología y la Educación Ambiental y II Congreso Nacional de Investigación en la

\section{Enseñanza de la Biología}

de la ganadería. De los encuestados el $80 \%$ viven en veredas pertenecientes al municipio de Tena (Laguneta y Catalamonte), el $20 \%$ restante viven en veredas de otros municipios. Estos aspectos, actividad económica y vivienda, son importantes pues por tradición o pertenencia, es más probable que la comunidad participe de colectivos para conservar dichos lugares, así fueran esporádicos o errantes.

La cobertura de servicios públicos, es en general deficiente, evidente en porcentajes bajos como el $30 \%$ de cobertura en Energía eléctrica, $24 \%$ en Acueducto, $19 \%$ Gas y $16 \%$ Teléfono. Estas deficiencias, obligan a los habitantes a realizar actividades alternativascomo: "abastecerse para cocinar directamente de los ríos, quebradas o nacimientos" en un 33\%, también la quema o entierro de basuras para quienes no cuentan con el servicio de recolección en un $42 \%$ y la utilización de leña para cocinar en un $25 \%$, las cuales generan un impacto negativo en el ecosistema, en los recursos naturales y de este modo en el bienestar de la comunidad, evidentes en la contaminación del agua, disminución de bosques por tala indiscriminada y aumento de enfermedades. En cuanto a la cobertura en salud, el $91 \%$ de los encuestados cuenta con servicio médico, éste último aspecto fundamental, pues los programas del sector salud son claves para lograr adecuados desempeños en los estudiantes (Choque, 2009).

En la categoría de educación, el $55 \%$ cursó la primaria, el $27 \%$ no culminó estudios primarios y sólo un $18 \%$ cursó algún grado en educación secundaria, ninguno tiene educación a nivel superior (técnico, tecnólogo o profesional). La población en general tiene un nivel educativo bajo, éste aspecto está estrechamente relacionado con las actividades económicas y la cobertura de servicios públicos, ya que la población rural se caracteriza por tener bajas condiciones de bienestar (ausencia de servicios, de infraestructura básica, etc.) y comparte condiciones culturales atrasadas (analfabetismo, etc.), como consecuencia de la dispersión y el aislamiento de sistemas sociales urbanos (Bustos, 2009), sumado a esto, los encuestados en general realizan actividades que no involucran un alto grado de alfabetización, lo cual fue un factor influyente en el desenvolvimiento actitudinal de los estudiantes, evidente en la falta de interés de niños y niñas por aprender y estudiar para mejorar sus condiciones de vida (Lewis, 2006).

En cuanto a la concepción ambiental, la relación que establecen los pobladores con los recursos naturales, es utilitarista donde la naturaleza es fuente de recursos que brindan o representan algún beneficio económico (cultivos, animales de producción, pastizales y agua), siendo una corriente opuesta al preservacionismo (Política Nacional de Biodiversidad, 1995).

En síntesis, hacer una lectura del contexto de los padres nos permite conocer el mundo simbólico de los estudiantes para recrear a posteriori estrategias 
Bio-grafia Escritos sobre la Biologia y su Enseñanza.

Edición Extra-Ordinaria. ISSN 2027-1034 P.p183-195

Memorias del VII Encuentro Nacional de Experiencias en la Enseñanza de la

Biología y la Educación Ambiental y II Congreso Nacional de Investigación en la

\section{Enseñanza de la Biología}

apropiadas en el desarrollo de propuestas en Educación Ambiental, razón por la que fueron importantes todas las categorías incluidas en la encuesta, dentro de las que sobresalen el nivel educativo, la categoría ocupacional y los ingresos de los progenitores, que en conjunto conforman la estructura financiera y cultural con la que cuentan los niños/as y que serán determinantes para acceder a otros niveles educativos e introducirse en el mercado laboral (Mediavilla y Calero, 2009). Así, son las actitudes que los padres poseen respecto a la educación y a la escuela del hijo las que tienen un efecto decisivo sobre el mismo, si los padres no valoran el aprendizaje, los hijos difícilmente lo harán (Sánchez, 2006) por lo que los estudiantes se benefician de padres informados, con actitudes positivas hacia la escuela, altas aspiraciones hacia los hijos y conductas positivas, en su educación (Georgiou, 1996).De esta forma, la familia juega un papel importante en el contexto de aprendizaje y afecta las experiencias de enseñanza de sus miembros (Gilet al., 1999) por ser la primera institución que ejerce influencia en el niño, que transmite valores, costumbres y creencias por medio de la convivencia diaria (Guevara, 2001), siendo clave el acompañamiento y la unión familiar, no solo para el rendimiento escolar sino para la formación de seres autónomos y responsables frente al ambiente, pues, a través de la interacción con sus hijos, los padres proveen experiencias que pueden influir positiva 0 negativamente en el crecimiento y desarrollo del niño (Korkatsch, 1998).

En la lectura de contexto para los estudiantes, la toma de datos fue reforzada con registros auditivos y visuales con sus transcripciones y grupos de discusión, por ser un grupo heterogéneo en edades. La Escuela Catalamonte cuenta con una población de 41 estudiantes, el $54 \%$ pertenecen a los grados $3^{\circ}, 4^{\circ}$ y $5^{\circ}$ y el $46 \%$ restante está conformado por los estudiantes más pequeños de grado $0^{\circ}$ a $2^{\circ}$, según la relación de matrícula 2009, organizados en "unidades multigrado"(clases con diferentes grados/cursos), también llamada organización horizontal, en donde se buscadistribuir al alumnado entre el profesorado disponible cuando se necesita hacer más de un grupo por curso. La encuesta se aplicó a 19 estudiantes con edades entre los 8 y 15 años, una cohorte de edad heterogénea que se distribuye sólo en los grados $3^{\circ}$ a $5^{\circ}$. Las edades que predominan son once años en un $32 \%$ y nueve en un $26 \%$, los estudiantes con doce y trece años están representados cada uno en un 11\%, la edad de ocho en un 10\% y sólo dos estudiantes representan cada uno un 5\% las edades de 10 y 15 años.

Respecto al núcleo y acompañamiento familiar, el 33\% de los estudiantes viven con su madre, el 39\% con ambos (padre y madre), el 28\% restante, son casos particulares que viven con el padre, o los abuelos, o están a cargo de bienestar familiar. La mayoría de los estudiantes (79\%) tienen hermanos en la escuela, el $54 \%$ tiene por lo menos uno, el $33 \%$ dos y un $13 \%$ tres hermanos, el $21 \%$ restante no tiene hermanos. De acuerdo con lo anterior, conservar un núcleo familiar primario no intervenido conformado por padres e hijos, puede tener mayor 
Memorias del VII Encuentro Nacional de Experiencias en la Enseñanza de la

Biología y la Educación Ambiental y II Congreso Nacional de Investigación en la

\section{Enseñanza de la Biología}

incidencia en el rendimiento escolar y sobretodo en el comportamiento y la expresión de un sistema de valores durante la realización de las actividades que implicó ésta investigación, ya que la familia es clave para tener estudiantes dispuestos emocionalmente para el logro de objetivos educativos (Choque, 2009), de forma particular, se mostraban más reacios o dispersos frente a las actividades aquellos niños y niñas que vivían con padrastros, abuelos (as), tíos u otros familiares, en vez de sus padres, pues no tenían el mismo nivel de exigencia y autoridad como los demás, o no recibían la atención suficiente.En cuanto a la tenencia de hermanos o no, de forma específica en el mismo salón, es un aspecto que potencializa la ayuda mutua mediante la "tutoría entre iguales" posibilitando una mayor participación del alumnado que "no sabe" y una mejor reelaboración del conocimiento del que "sabe más", siendo éste último otra de las características de la heterogeneidad según Iglesias (2008).

De los estudiantes, el $89 \%$ no trabajan, mientras el 11\% sí lo hacen en labores del campo no remuneradas, relacionadas con pequeños cultivos o producción animal no tecnificada, cacería y domesticación de animales silvestres para su venta como mascotas, pues la transmisión de conocimientos se presenta mediante procesos de socialización primaria vinculando a los niños/as en las actividades de la vida adulta a través de la convivencia en familia, grupos de trabajo y producción (Díaz, 2005), otros ayudan a sus padres que se emplean en restaurantes o comercio. Si los estudiantes son económicamente activos en sus hogares es un aspecto importante que influye en su rendimiento escolar y que está relacionado también con dificultades para completar los estudios (bajo rendimiento o deserción) al combinar actividades escolares con trabajo doméstico, remunerado 0 no. (Choque, 2009)

En cuanto a la percepción de ambiente, las respuestas pueden agruparse en Elementos yAcciones, en la primera están los lugares, las personas, las cosas y los seres de la naturaleza, elementos tangibles e intangibles, mientras que la segunda categoría consiste en el comportamiento o las intenciones que muestran o realizan los estudiantes frente al medio ambiente, las cuales concuerdan con cuatro concepciones permeadas por la cultura y la educación para definir dinámicas y procesos específicos que otros conceptos o categorías no permiten, donde el ambiente es problema para solucionar, recurso para administrar, medio de vida y naturaleza para apreciar, respetar y preservar (Duarte, 2003).

\section{Conclusiones.}

-Una propuesta en Educación Ambiental para el conocimiento y conservación de la avifauna, debe fortalecer compromisos de cambio acordes con las necesidades de apertura a las diferentes formas de expresión de la vida local y de la cultura de los pobladores que configuran dicha región, de modo que esta investigación incluyó experiencias y herramientas de aprendizaje que orientaron a maestros, 
Memorias del VII Encuentro Nacional de Experiencias en la Enseñanza de la

Biología y la Educación Ambiental y II Congreso Nacional de Investigación en la

\section{Enseñanza de la Biología}

estudiantes y habitantes, a conocer la avifauna local y a incluirla como un instrumento de conservación en diferentes contextos.

-El saber o conocimiento de las comunidades, sus prácticas y alcances que se perpetúan con el tiempo a través de la cultura, y las relaciones que se establecen con los demás, influidas por factores económicos, políticos, naturales y sociales deben ser la carta de navegación en propuestas orientadas hacia la conservación de las aves, para que sean ellos mismos quienes reconozcan su responsabilidad frente a una problemática ambiental específica como parte del problema y de la solución.

-Las personas aprenden en diferentes espacios, en la escuela, en procesos, instituciones, relaciones personales, recibiendo mensajes y propuestas, elaborando códigos, e interpretando normas sociales, conocimientos, creencias, valores, saberes, habilidades, aptitudes, competencias y sentimientos, por lo que el punto de partida de cualquier propuesta en Educación Ambiental debe ser el diagnóstico ambiental, para entender y conocer las interrelaciones que tejen los participantes con el medio, la visión, actuación y construcción de Ambiente desde su contexto, y así brindar un soporte real a la problemática ambiental.

- La lectura del contexto comprendió unidades con significado o categorías que indagaron aspectos de orden personal, social y ambiental, para legitimar los comportamientos que los participantes exteriorizan en cuanto al aprovechamiento de los recursos naturales, y un sistema de valores y actitudes que ponen frente a estos.

-Los determinantes internos y externos que ejercen influencia negativa o positiva en los comportamientos de los participantes y por ende en su aprendizaje, son la familia, su composición, el acompañamiento, el nivel educativo, su situación económica, la institución educativa, el entorno social y cultural.

\section{Bibliografía}

ÁNGEL Maya, Carlos Augusto (1995). La fragilidad ambiental de la cultura. Editorial Universidad Nacional: Instituto de Estudios Ambientales. IDEA.Colombia. BATTAHYÁNY, Karina y CABRERA, Mariana (coord.) (2011). Metodología de la investigación en ciencias Sociales. Apuntes para un curso inicial. Universidad de la República Uruguay. Montevideo.

BIRDLIFE (2005). Áreas Importantes para la Conservación de las Aves en los Andes Tropicales: sitios prioritarios para la conservación de la biodiversidad.Quito, Ecuador. 
Bio-grafia Escritos sobre la Biología y su Enseñanza.

Edición Extra-Ordinaria. ISSN 2027-1034 P. p183-195

Memorias del VII Encuentro Nacional de Experiencias en la Enseñanza de la Biología y la Educación Ambiental y 11 Congreso Nacional de Investigación en la Enseñanza de la Biología

BUSTOS, Antonio. (2009) Valoraciones del profesorado de Escuela Rural sobre el entorno presente.Revista Iberoamericana de Educación [http://www.rieoei.org/deloslectores/2763Bustosv2.pdf]

CEPEDA, Edilberto \& CAICEDO, Gloria (2007). Factores asociados a la calidad de la educación. Revista Iberoamericana de Educación [http://dialnet.unirioja.es/servlet/articulo?codigo=2325266]

CORPORACIÓN AUTÓNOMA REGIONAL DE CUNDINAMARCA (2003). Plan de Manejo Ambiental de los Humedales de Neuta, Laguna la Herrera y Laguna de Pedropalo.

CORBETTA, Piergiorgio (2003). Metodología y técnicas de la investigación social. Mc Graw Hill. México.

CHOQUE, Larrauri Raúl (2009). Ecosistema Educativo y Fracaso Escolar.Revista Iberoamericana de Educación [http://www.rieoei.org/2967.htm]

DELGADO, Juan Manuel y GUTIÉRREZ, Juan (Coord) (1999), Métodos y técnicas cualitativas de investigación en ciencias Sociales, Madrid, Síntesis.

DIAZ, Ruy (2005). Educación, cultura y supervivencia. Revista Iberoamericana de Educación. [http://www.rieoei.org/historia02.htm]

DUARTE, Jakeline (2003). Ambientes de Aprendizaje: una aproximación conceptual. Universidad de Antioquia. Revista Iberoamericana de Educación [http://www.rieoei.org/rec dist1.htm]

FREILE, J. F. y SANTANDER, T. (2005). Áreas Importantes para la Conservación de las Aves en los Andes Tropicales: sitios prioritarios para la conservación de la biodiversidad. Quito, Ecuador. Serie de Conservación de BirdLife No. 14.

GEORGIOU, S. (1996). Parental Involvement in Cyprus", en International Journal of Educational Research.

GIL, P.M, ROBLIZO, M.J., GÓMEZ I (1999). Actitud y contexto ante los quehaceres escolares: el estudio de un caso en primaria. Revista Iberoamericana de Educación [http://www.rieoei.org/rie45a09.htm]

GUEVARA NIEBLA, G. (1996). La relación familia-escuela. [http://www.rieoei.org/1538.htm] 
Memorias del VII Encuentro Nacional de Experiencias en la Enseñanza de la

Biología y la Educación Ambiental y II Congreso Nacional de Investigación en la Enseñanza de la Biología

GOYETE\&LESSARD, H. (1988). La investigación-acción. Funciones, fundamentos e instrumentos. Barcelona. Ediciones Laertes.

IGLESIAS, J.C. (2008). Agrupamiento flexible de alumnos en la Educación Primaria. Una respuesta a la diversidad.

KORKATSCH-GROSZKO, M (1998). How can Parents Assist in the Learning Process? What Classroom Teachers may Suggest. [http://ericir.syr.edu/plweb]

LEWIS, A. Helping Young Urban Parents Educate Themselves and their Children [Digests]. Disponible en: http://www.ed.gov/databases/ERIC Digests/ed355314.html.

MEDIAVILLA, Mauro y CALERO, Jorge (2009). Determinantes internos y externos en el proceso de aprendizaje: una aproximación al caso español a partir de la EVC-05. En: Revista lberoamericana de Educación[http://www.rieoei.org/deloslectores/3070Bordalejo.pdf]

MOLERIO, O., OTERO, I., NIEVES, Z (2007). Aprendizaje y desarrollo humano. Universidad Central "Marta Abreu" de las Villas, Cuba. En: Revista Iberoamericana de Educación[http://www.rieoei.org/deloslectores/1901Perez.pdf]

PEREDA, Carlos., PRADA, Miguel. y ATIS, Walter (2003).Investigación Acción Participativa: Propuesta para un ejercicio activo de la ciudadanía. Madrid, España: Colectivo loé. 2003. [http://www.colectivoioe.org/investigaciones articulos.php?op=articulo\&id=95]

PEREZ, S.G. (2004) Modelos de Investigación cualitativa en Educación Social y Animación socio-cultural, aplicaciones prácticas. Madrid, España. Ediciones Narcea, S.A.

POLÍTICA NACIONAL DE BIODIVERSIDAD, 1995.

RENJIFO L.M., FRANCO, A.M, AMAYA, J.D., KATTAN G.H., LÓPEZ B. (2002). Libro Rojo de Aves de Colombia. Instituto de Recursos Biológicos Alexander Von Humboldt y Ministerio del Medio Ambiente.

SANCHEZ, Escobedo Pedro (2006). Discapacidad, familia y logro escolar. México. En: Revista Iberoamericana de Educación [http://www.rieoei.org/1538.htm]

SAUTU Ruth, BONIOLO Paula, DALLE Pablo y EBERT Rodolfo. (2005). Manual de metodología. Construcción del marco teórico, formulación de los objetivos y elección de la metodología. El estudio de las interpretaciones que hacen las personas de la realidad social no utiliza exclusivamente metodologías cualitativas. Consejo Latinoamericano de Ciencias Sociales. 
Bio-grafia Escritos sobre la Biología y su Enseñanza.

Edición Extra-Ordinaria. ISSN 2027-1034 P.p183-195

Memorias del VII Encuentro Nacional de Experiencias en la Enseñanza de la Biología y la Educación Ambiental y II Congreso Nacional de Investigación en la Enseñanza de la Biología

TORRES, Maritza (2002). Incorporación de la dimensión ambiental en zonas rurales y pequeño urbanas del país. Convenio MEN - MINAMBIENTE (Crédito BID). Bogotá, Colombia. 